\title{
Backward-Forward Search for Manipulation Planning
}

\author{
Caelan Reed Garrett, Tomás Lozano-Pérez, and Leslie Pack Kaelbling
}

\begin{abstract}
In this paper we address planning problems in high-dimensional hybrid configuration spaces, with a particular focus on manipulation planning problems involving many objects. We present the hybrid backward-forward (HBF) planning algorithm that uses a backward identification of constraints to direct the sampling of the infinite action space in a forward search from the initial state towards a goal configuration. The resulting planner is probabilistically complete and can effectively construct long manipulation plans requiring both prehensile and nonprehensile actions in cluttered environments.
\end{abstract}

\section{INTRODUCTION}

Many of the most important problems for robots require planning in high-dimensional hybrid spaces that include both the state of the robot and of other objects and aspects of its environment. Such spaces are hybrid in that they involve a combination of continuous dimensions (such as the pose of an object, the configuration of a robot, or the temperature of an oven) and discrete dimensions (such as which object(s) a robot is holding, or whether a door has been locked).

Hybrid planning problems have been formalized and addressed in the robotics literature as multi-modal planning problems and several important solution algorithms have been proposed [1], [2], [3], [4], [5], [6], [7]. A critical problem with these algorithms is that they have relatively weak guidance from the goal: as the dimensionality of the domain increases, it becomes increasingly difficult for a forward-search strategy to sample effectively from the infinite space of possible actions.

In this paper, we propose the hybrid backward-forward (HBF) algorithm. Most fundamentally, HBF is a forward search in state space, starting at the initial state of the complete domain, repeatedly selecting a state that has been visited and an action that is applicable in that state, and computing the resulting state, until a state satisfying a set of goal constraints is reached. However, a significant difficulty is that the branching factor is infinite- there are generally infinitely many applicable actions in any given state. Furthermore, random sampling of these actions will generally not suffice: actions may need to be selected from very small or even lower-dimensional subspaces of the space of applicable actions, making it a measure 0 event to hit

Computer Science and Artificial Intelligence Laboratory, Massachusetts Institute of Technology, Cambridge, MA 02139 USA caelan@mit.edu, tlp@mit.edu, lpk@mit.edu

This work was supported in part by the NSF (grant 1420927). Any opinions, findings, and conclusions or recommendations expressed in this material are those of the author(s) and do not necessarily reflect the views of the National Science Foundation. We also gratefully acknowledge support from the ONR (grant N00014-14-1-0486 ), from the AFOSR (grant FA23861014135), and from the ARO (grant W911NF1410433).

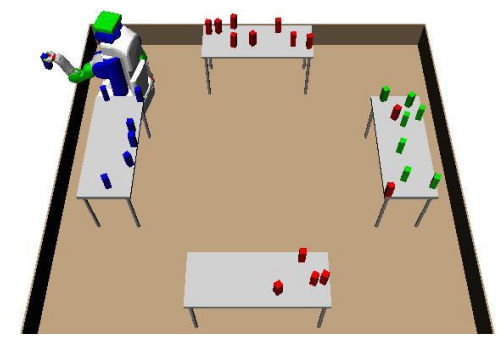

Fig. 1: A near-final state of a long-horizon problem.

an appropriate one at random. For example, consider the problem of moving the robot base to some configuration that may be the initial step of a plan to pick up and move an object. That configuration has to be selected from the small subset of base poses that have an inverse-kinematics solution that allows it to pick up the object. Or, in trying to select the grasp for an object, it may be critical to select one in a smaller subspace that will allow the object to be subsequently used for a task or placed in a particular pose.

HBF solves the exact planning problem by strongly focusing the sampling of actions toward the goal. It uses backward search in a simplified problem space to generate sets of actions that are useful: these useful actions are components of successful plans in the simplified domain and might plausibly be contained in a plan to reach the goal in the actual domain. The backward search constructs a reachability graph, working backward from the goal constraints and using them to drive sampling of actions that could result in states that satisfy them. In order to to make this search process simpler than solving the overall problem, the constraints in the final goal, as well as constraints that must hold before an action can be applied, are considered independently. Focusing the action sampling in this way maintains completeness while providing critical guidance to the search for plans in highdimensional domains.

\section{A. Related work}

Our work draws from existing approaches to robot manipulation planning, integrated task and motion planning, and symbolic AI planning.

In manipulation planning, the objective is for the robot to operate on objects in the world. The first treatments considered a continuous configuration space of both object placements and robot configurations, but discrete grasps [8], [9], [10]; they were more recently extended to selecting from a continuous set of grasps and formalized in terms of a manipulation graph [11], [12]. The approach was extended to complex problems with a single movable object, possibly 
requiring multiple regrasps, by using probabilistic roadmaps and a search decomposition in which a high-level sequence of transit and transfer paths is first identified, and then motion planning attempts to achieve it [1].

Hauser [3], [4] identified a generalization of manipulation planning as multi-modal planning, that is, planning for systems with multiple (possibly infinitely many) modes, representing different constraint sub-manifolds of the configuration space. Plans alternate between moving in a single mode, where the constraints are constant, and switching between modes. Hauser provided an algorithmic framework for multi-modal planning that is probabilistically complete given effective planners for moving within a mode and samplers for transitioning between modes. Barry et al. [6] addressed larger multi-modal planning problems using a bidirectional RRT-style search, combined with a hierarchical strategy, similar to that of HBF, of suggesting actions based on simplified versions of the planning problem.

Many recent approaches to manipulation planning integrate discrete task planning and continuous motion planning algorithms; they pre-discretize grasps and placements so that a discrete task planner can produce candidate highlevel plans, then use a general-purpose robot motion planner to verify the feasibility of candidate task plans on a robot [13], [14], [15], [16]. Some other systems combine the task planner and motion planner more intimately; although they generally also rely on discretization, the sampling is generally driven by the task [2], [17], [18], [19], [7], [20].

Effective domain-independent search guidance has been a major contribution of research in the artificial intelligence planning community, which has focused on state-space search methods; they solve the exact problem, but do so using algorithmic heuristics that quickly solve approximations of the actual planning task to estimate the distance to the goal from an arbitrary state [21], [22]. One effective approximation is the "delete relaxation" in which it is assumed that any effect, once achieved by the planner, can remain true for the duration of the plan, even if it ought to have been deleted by other actions [23]; the length of a plan to achieve the goal in this relaxed domain is an estimate that is the basis for the $\mathrm{H}_{\mathrm{FF}}$ heuristic.

The most closely related approach to $\mathrm{HBF}$ integrates symbolic and geometric search into one combined problem and provides search guidance using an adaptation of the $\mathrm{H}_{\mathrm{FF}}$ heuristic to directly include geometric considerations [20]. It was able to solve larger pick-and-place problems than most previous approaches but suffered from the need to pre-sample its geometric roadmaps.

\section{B. Problem formulation}

Our objective is to find a feasible plan in a hybrid configuration space. A configuration variable $V_{i}$ defines some aspect or dimension of the overall system; its values may be drawn from a discrete or continuous set. A configuration space $\mathcal{S}$ is the Cartesian product of the domains of a set of configuration variables $V_{1}, \ldots, V_{n}$. A state $s=\left\langle v_{1}, \ldots, v_{n}\right\rangle \in \mathcal{S}$ is an element of the configuration space consisting of assignments of values to all of its configuration variables.

A simple constraint $C_{i}$ is a restriction on the possible values of state variable $i$ : it can be an equality $V_{i}=C_{i}$, or a set constraint $V_{i} \in C_{i}$, where $C_{i}$ is a subset of the domain of $V_{i}$. A constraint $C_{i_{1}, \ldots, i_{k}}$ is a restriction on the possible values of a set of state variables $V_{i_{1}}, \ldots, V_{i_{k}}$, constraining $\left(V_{i_{1}}, \ldots, V_{i_{k}}\right) \in C_{i_{1}, \ldots, i_{k}}$, where $C_{i_{1}, \ldots, i_{k}}$ is a subset of the Cartesian products of the domains of $V_{i_{1}}, \ldots, V_{i_{k}}$. We will assume that there is a finite set of constraint types $\xi \in \Xi$; each $\xi$ specifies a constraint using a functional form so that $C_{i_{1}, \ldots, i_{k}}$ holds whenever $\xi\left(V_{i_{1}}, \ldots, V_{i_{k}}\right)=0$.

A state $s=\left\langle v_{1}, \ldots, v_{n}\right\rangle$ satisfies a constraint $C_{i_{1}, \ldots, i_{k}}$ if and only if $\left\langle v_{i_{1}}, \ldots, v_{i_{k}}\right\rangle \in C_{i_{1}, \ldots, i_{k}}$. An effect $E_{i}$ is an equality constraint on $V_{i}$, used to model the effect of taking an action on that variable.

An action $a$ is an effector primitive that may be executed by the robot. It is characterized by a set of condition constraints $C^{1}, \ldots, C^{K}$ on some subset of the the configuration variables (written a.con), and a set of effects $E^{1}, \ldots, E^{l}$ on some generally distinct set of configuration variables. For any state $s=\left\langle v_{1}, \ldots, v_{n}\right\rangle$ that satisfies constraints $C^{1}, \ldots, C^{K}$, in which the robot executes action $a$, the resulting state a.eff $(s)$ has $V_{i}=v_{i}$ for all configuration variables not mentioned in the effects, and value $V_{I\left(E^{j}\right)}=E^{j}$ for all configuration variables in the effects set. (We use $I(E)$ to denote the index of some particular effect). A planning problem is an initial state $s_{0} \in \mathcal{S}$ and goal set of constraints $\Gamma$. A plan is a sequence $a_{1}, \ldots, a_{m}$ of actions; it solves a planning problem $s_{0}, \Gamma$ if $a_{m}$.eff $\left(\ldots a_{1}\right.$.eff $\left.\left(s_{0}\right)\right) \in \Gamma$.

\section{HBF ALGORITHM}

In this section, we describe the HBF algorithm in detail, beginning with the top-level forward search, continuing with the definition of a reachability graph, then showing how focused action sampling and a heuristic can be derived from the reachability graph.

\section{A. Forward search: persistent enforced hill-climbing}

Figure 2 shows a basic framework for persistent enforced hill-climbing; we use this form of search because it can maintain completeness with infinite action spaces and because attempts to find a path of optimal length can quickly become bogged down with a large agenda. It is given as input an initial state $s_{0}$, a set of goal constraints $\Gamma$, and a set of possible actions $\mathcal{A}$. It keeps a queue of search nodes, each of which stores a state $s \in \mathcal{S}$, an action $a \in \mathcal{A}$, and a pointer to its parent node in the tree. A heuristic function $\mathrm{H}$, which we discuss in section II-C, is used to compute an estimate of the distance to reach a state satisfying $\Gamma$ from state $s$. The search enforces hill-climbing, by remembering the minimum heuristic value $h_{\min }$ seen so far. Whenever a state is reached with a lower heuristic value, the entire queue is popped, leaving only the initial state (in case this branch of the tree is a dead-end) and the current state on the queue. If the queue has not been reset, it adds the node that it just popped off back to the end of the queue. This last 


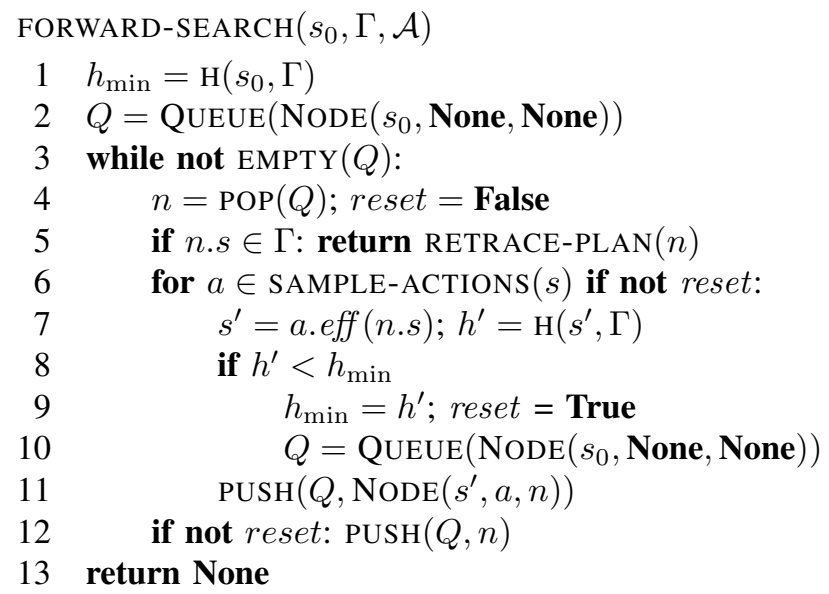

Fig. 2: Top-level forward search.

step is critical for achieving completeness in domains with an infinite action space: it ensures that we consider taking other actions in the state associated with that node.

Intuitively, then, whenever a state with an improved heuristic value is reached, the queue is left containing just $s_{0}$ and the most recent state (call it $s$ ). Then, until a better state is reached, we will: sample a child $s_{0}^{1}$ of $s_{0}$ (now the queue is: $s, s_{0}^{1}, s_{0}$ ); sample a child $s^{1}$ of $s$ (now the queue is: $s_{0}^{1}, s_{0}$, $s^{1}, s$ ), sample a child $s_{0}^{(1,1)}$ of $s_{0}^{1}$ (now the queue is $s_{0}, s^{1}$, $s, s_{0}^{(1,1)}, s_{0}^{1}$ ), sample a new child $s_{0}^{2}$ of $s_{0}$ (now the queue is $\left.s^{1}, s, s_{0}^{(1,1)}, s_{0}^{1}, s_{0}^{2}, s_{0}\right)$, and so on. This search strategy is as driven by the heuristic as possible, but takes care to maintain completeness through the "persistent" sampling at different levels of the search tree.

We compactly characterize the set of possible actions using action templates with the following form:

ACTIONTEMPLATE $\left(\theta_{1}, \ldots, \theta_{k}\right)$ :

$$
\begin{array}{ll}
\text { con }: & \left(q_{i_{1}}, \ldots, q_{i_{t}}\right) \in C_{i_{1}, \ldots, i_{t}}(\theta) \\
& \ldots \\
\text { eff }: & q_{j}=e_{j}(\theta)
\end{array}
$$

An action template specifies a generally infinite set of actions, one for each possible binding of the parameters $\theta_{i}$, which may be drawn from the domains of state variables or other values such as object designators. We assume that action templates are never instantiated with a set of parameters that violate permanent constraints (e.g., exceeding joint limits or colliding with immovable objects objects).

In addition to the heuristic, we have also left indeterminate how an action or set of actions is chosen to be applied to a newly reached state $s$. To do so, we must introduce the notion of a reachability graph.

\section{B. Reachability graphs}

A reachability graph $(\mathrm{RG})$ is an oriented hyper-graph in which: (1) each vertex is a point in (a possibly lowerdimensional subspace of) the configuration space $\mathcal{S}$, which can be represented as an assignment of values to a set of configuration variables $\left\{V_{i_{1}}=v_{i_{1}}, \ldots, V_{i_{k}}=v_{i_{k}}\right\}$; (2) each edge is labeled with an action $a$; (3) the outgoing vertices of the edge are the effects of $a$; and (4) the incoming vertices of the edge are assignments that collectively satisfy the condition constraints of $a$.

We say that an RG $G$ contains a derivation of constraint $C$ from initial state $s$ if either (1) $C$ is satisfied in $s$ or (2) there exists an action $a \in G$ that has $C$ as an effect and for all $C_{i} \in A$.con, $G$ contains a derivation of $C_{i}$ from $s$.

To construct the RG, we solve a version of the planning problem that is simplified in multiple ways, allowing very efficient identification of useful actions. There are three significant points of leverage: (1) individual constraints in the goal and in the conditions for an action are solved independently; (2) it is possible to plan efficiently in lowdimensional subspaces (for example, by using an RRT to find a path for the robot alone, or for an object alone); and (3) plans made in low-dimensional subspaces are allowed to violate constraints from the full space (for example, colliding with an object) and the system subsequently plans to achieve those constraints that were violated (for example, by moving the object that cause the collision out of the way).

The fundamental organizing idea is the constrained operating subspace (COS). A COS is a subspace of $\mathrm{S}$ defined by two restrictions: first, it is restricted to a set of operating constraint variables (OCV's), and then further restricted to the intersection of that space with a set of constraints $\mathcal{C}$ that are defined in terms of the OCV's. A COS provides methods, used in planning, for generating potential samples in that subspace and also for generating action instances to move into and within the subspace. Every $\cos \Omega$ is defined by:

- A set $\mathcal{V}=\left\{V_{i_{1}}, \ldots, V_{i_{k}}\right\}$ of configuration variables;

- A set $\mathcal{C}$ of constraints;

- An intersection sampler, which generates samples from $\mathcal{C} \cap C_{i}$ for some domain constraint $C_{i}$ of one of the functional forms in $\Xi$;

- A transition sampler, which generates samples $\left(o, a, o^{\prime}\right)$, where $o^{\prime}=\left\langle v_{i_{1}}, \ldots, v_{i_{k}}\right\rangle \in \Omega, a$ is an action whose effects include (but need not be limited to) $V_{i_{1}}=v_{i_{1}}, \ldots, V_{i_{k}}=v_{i_{k}}$, and $o$ is an element of a different COS.

- A roadmap sampler, which generates samples $\left(o, a, o^{\prime}\right)$ where $o \in \Omega, o^{\prime} \in \Omega$, and $a$ is an action such that the initial abstract state $o$ satisfies the condition constraints of $a$ that apply to $\Omega$ 's variables and the effects of $a$ include $o^{\prime}$.

Planning within a COS is completely restricted to that subspace, observing only constraints involving values of variables in $\mathcal{V}$. Our definition of a $\cos$ is in contrast to Hauser's definition of a mode, in that Hauser's modes are defined on the complete configuration space, so that every variable either is fixed or can be controlled by the system.

In a domain with infinite state and action spaces, the corresponding RG will generally be infinite as well. We will iteratively construct a subgraph of the full RG that can derive a set of goal constraints $\Gamma$ from a state $s$. The RG subgraph will eventually contain actions that are the first step of a 


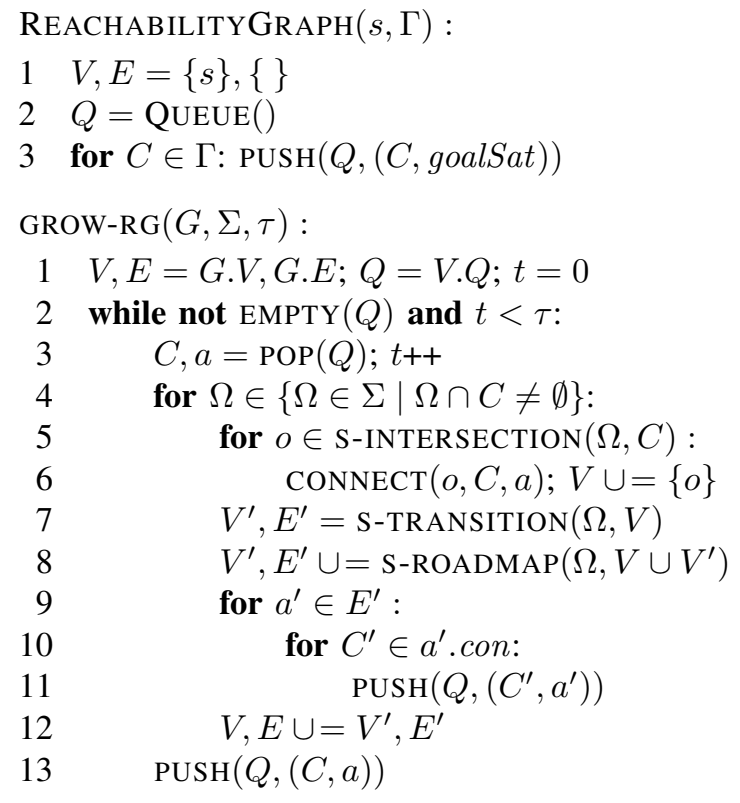

Fig. 3: Construction and growth of the reachability graph.

solution to the planning problem $(s, \Gamma)$. Because it considers the satisfaction of constraints independently, it may be that there are actions that produce all of the condition constraints of some other action, but those actions are incompatible in a way that implies that this collection of actions could not, in fact, be sequenced to provide a solution. For this reason, during the forward search, we will return to the process of growing the RG, until it generates actions that are, in fact, on a feasible plan.

Procedure REACHABILITYGRAPH creates a new RG, with start state $s$ as a vertex (meaning that the assignment of values to variables in $s$ can be used to satisfy conditions of edges that are added to the RG) and adds an agenda item for each constraint in the goal; these constraints are associated with the dummy action goalSat, which, when executable implies that the overall relaxed goal is satisfied.

Procedure GROwRG takes as input a partial RG $G$ (either just initialized or already previously partially grown), a set of COS's $\Sigma$, and a time-out parameter $\tau$. We assume that it, as well as all of the COS samplers in $\Sigma$, have a set of action templates $\mathcal{A}$ available to them. It grows the RG for $\tau$ steps by popping a $C, a$ pair off of the queue, and trying to find actions that can help achieve $C$ and adding them to the RG. It begins by generating sample elements $O$ of the intersection between $\Omega$ and $C$ using S-INTERSECTION. It adds them to the set of vertices and connects them as "witnesses" that can be used to satisfy the $C$ condition of action $a$. Now the transition sampler (S-TRANSITION) seeks $\left(o, a^{\prime}, o^{\prime}\right)$ triples, where $o \in \Omega, o^{\prime} \in \Omega^{\prime}$, and $o$ reaches $o^{\prime}$ via action $a^{\prime}$. Finally, S-ROADMAP samples $\left(o, a^{\prime}, o^{\prime}\right)$ triples and adds vertices and hyperedges that move within $\Omega$. Each condition of each new edge is added to the queue, and the original node is placed back in the queue for future expansion.

\section{Action sampling and heuristic}

Now, we can revisit the forward search algorithm in figure 2 to illustrate the points of contact with the backward algorithm. The RG for $s$ is first used to compute its heuristic value. The heuristic procedure $\mathrm{H}$ initializes an $\mathrm{RG}$ for $(s, \Gamma)$ if necessary; if the RG does not yet contain a derivation for all the constraints in $\Gamma$ from $s$, then the graph is grown in an attempt to find such a derivation. A heuristic estimate of the distance to reach $\Gamma$ from $s$ is the number of actions in a derivation. Because computing the minimum length derivation is NP-hard, we use the $\mathrm{H}_{\mathrm{FF}}$ algorithm which greedily chooses a small derivation [23]. Because the RG and relaxed plan graph of the $\mathrm{H}_{\mathrm{FF}}$ algorithm both make a similar independence approximation, $\mathrm{H}_{\mathrm{FF}}$ can be computed on top of the RG with minimal algorithmic changes.

The SAMPLEACTIONS procedure seeks actions from the RG that are applicable in $s$ but have not yet been applied. If there are no such actions, then the RG is grown for $\tau$ steps. In our experience, it is frequently the case that as soon as a derivation is found, there is at least one action in the RG that is feasible for use in forward search. To increase performance in practice, the first set of actions returned are restricted to the helpful actions computed by the $\mathrm{H}_{\mathrm{FF}}$ algorithm [23].

HBF can be shown to be probabilistically complete if the COS samplers are themselves probabilistically complete. The intuition behind the argument is that the reachability graph constructs a superset of the set of solutions, due to its independence approximation. The proof is similar in nature to the probabilistic completeness proof by Hauser [4]. Due to space limitations, a sketch of this argument can be found at the following URL: http://web.mit.edu/caelan/ WWW/research/hbf/

\section{DOMAIN AND EXAMPLE}

In this section, we describe aspects of the domain formalization used in the experiments of the last section and work through an illustrative example. HBF is a generalpurpose planning framework for hybrid domains; here we are describing a particular instance of it for mobile manipulation.

Our domain includes a mobile-manipulation robot (based on the Willow Garage PR2) that can pick up, place, and push small rigid objects. The configuration spaces for problem instances in this domain are defined by the following configuration variables: $r$ is the robot's configuration, $o_{i}$ is the pose of object $i, h$ is a discrete variable representing which object the robot is holding (its value is the object's index or NONE), and $g$ is the grasp transform between the held object and the gripper or NONE.

\section{A. Action templates}

The robot may move from configuration $q$ to configuration $q^{\prime}$ if it is not holding any object and there is a collision-free trajectory, $\tau$, from $q$ to $q^{\prime}$ given the poses of all the objects. Each object $i$ also has a constraint that its pose $o_{i}$ be in the set of poses that do not collide with $\tau$. This set is represented by C-FREE-POSES $i$. 
$\operatorname{Move}\left(q, q^{\prime}, \tau\right)$ :

$$
\begin{array}{ll}
\text { con }: & r, h=q, \text { None } \\
& o_{i} \in \mathrm{C} \text {-FREE-POSES } \\
i & (\tau) \forall i \\
\text { eff }: & r=q^{\prime}
\end{array}
$$

If the robot is holding object $j$ in grasp $\gamma$, it may move from configuration $q$ to $q^{\prime}$; the free-path constraint is extended to include the held object and the effects are extended to include the fact that the pose of the held object changes as the robot moves. The values of $r$ and $o_{i}$ change in such a way that $g$ is held constant.

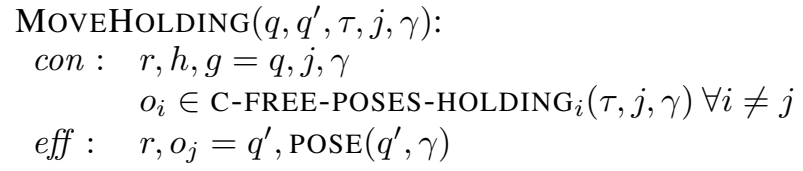

The PICK action template characterizes the state change between the robot holding nothing and the robot holding (being rigidly attached to) an object. It is parameterized by a robot configuration $q$, an object to be picked $j$, and a grasp transformation $\gamma$. If the robot is in the appropriate configuration, the hand is empty, and the object is at the pose obtained by applying transformation $\gamma$ to the robot's end-effector pose when it is in configuration $q$, then the grasp action will succeed, resulting in object $j$ being held in grasp $\gamma$. No other variables are changed. The PLACE template is the reverse of this action. UNSTACK and STACK action templates can be defined similarly by augmenting PICK and PLACE with a parameter and constraint involving the base object.

$$
\begin{aligned}
& \operatorname{PICK}(q, j, \gamma) \text { : } \\
& \text { con: } r, h=q \text {, None } \\
& o_{j}=\operatorname{Pose}(q, \gamma) \\
& \text { eff : } \quad h, g=j, \gamma \\
& \begin{array}{cl}
\operatorname{Place}(q, j, \gamma): \\
\text { con }: \quad r, h, g=q, j, \gamma \\
\text { eff }: \quad h=\text { None } \\
& o_{j}=\operatorname{Pose}(q, \gamma)
\end{array}
\end{aligned}
$$

The Push action is similar to MoveHolding except that grasp transformation parameter $g$ is replaced with an initial pose parameter $p$ for the object. Procedure END-POSE $j$ computes the resulting pose of the object if the robot moves from $q$ to $q^{\prime}$ along a straight-line path.

$$
\begin{aligned}
& \operatorname{Push}\left(q, q^{\prime}, j, p\right) \text { : } \\
& \text { con: } r, h, o_{j}=q \text {, None, } p \\
& o_{i} \in \text { C-FREE-POSES-PUSH }{ }_{i}\left(q, q^{\prime}, j, p\right) \forall i \neq j
\end{aligned}
$$

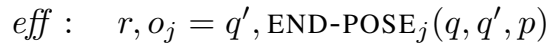

\section{B. Constrained operating subspaces}

Action templates define the fundamental dynamics of the domain; but to make HBF effective, we must also define the COSs that will be used to construct RGs, selection actions, and compute the heuristic.

- $\Omega_{\text {move }}$ considers the robot configuration, $\mathcal{V}=\{r\}$, and has no constraints.

- $\Omega_{\text {moveHolding }(i, g)}$ is parameterized by an object $i$ and grasp $g$; it considers the robot configuration and pose of object $I, \mathcal{V}=\left\{r, o_{i}\right\}$ subject to the grasp constraint $\mathcal{C}=\left\{o_{i}=\operatorname{POSE}(r, g)\right\}$.

- $\Omega_{\text {push }(i)}$ is parameterized by an object $i$; it abstracts away from the robot configuration and considers only the object's pose, $\mathcal{V}=\left\{o_{i}\right\}$, subject to the constraint

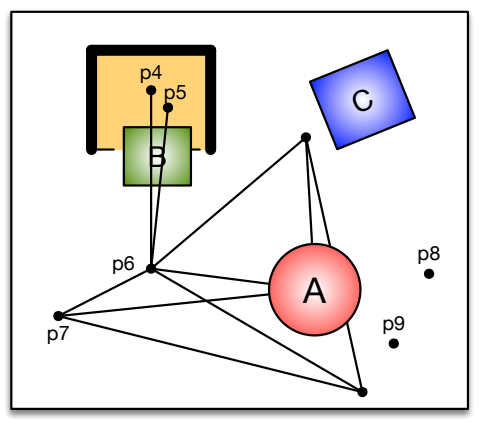

Fig. 4: An example initial state, with roadmap for object $A$.

that $o_{i} \in$ table (where table is the space of poses for $o_{i}$ for which it is on the table.)

- $\Omega_{\text {place }(i)}$ is parameterized by object $i$ for graspable objects; it also considers only the object's pose, $\mathcal{V}=$ $\left\{o_{i}\right\}$, subject to the constraint $\mathcal{C}=\left\{o_{i} \in\right.$ table $\}$.

- $\Omega_{\text {pick(i) }}$ has the same parameters, $\mathcal{V}$ and $\mathcal{C}$ as $\Omega_{\text {place }(i)}$.

Each of these COS's is augmented with sampling methods. Placements on flat surfaces and other objects, as well as placements in regions, are generated using Monte Carlo rejection sampling. Inverse reachability and inverse kinematics sample robot configurations for manipulator transforms. The S-ROADMAP samplers for moving and moving while holding are bidirectional RRT's that plan for the robot base then robot arm. The S-ROADMAP sampler for pushing is also a bidirectional RRT in the configuration space of just an object. Each edge of the tree is an individual push action computed by a constrained workspace trajectory planner that samples a robot trajectory that can perform the push.

\section{Example}

Figure 4 shows a top-down view of a table with three movable objects and a fixed u-shaped obstacle. This is the initial state $s_{0}$ of a planning problem for which the goal $\Gamma=\left\{o_{A} \in R\right\}$, where $R$ is the region of configuration space for object $A$ that would place it on the table within the fixed obstacle. Assuming that the robot is in some initial configuration $q_{0}$, we can describe $s_{0}$ as $\left\langle r=q_{0}, o_{A}=\right.$ $p_{1}, o_{B}=p_{2}, o_{C}=p_{3}, h=$ none, $g=$ none $\rangle$. Objects $B$ and $C$ can be grasped but $A$ cannot.

To begin the forward search, HBF must construct an initial $\mathrm{RG}$, which will be used to compute the initial heuristic value $h_{\min }$ and to generate the first set of actions in the search. In the following, we will sketch the process by which the RG, $G$, is constructed, showing parts of $G$ in figure 5 We add $s_{0}$ to the set of vertices, add a dummy action with $\Gamma$ as its conditions constraints to the set of edges, and initialize $Q=\left[\left(o_{A} \in R\right.\right.$, goalSat $\left.)\right]$.

Iteration 1: $C, a=\left(o_{A} \in R\right.$, goalSat $)$ is popped from $Q$. The only $\Omega$ that has a non-null intersection with $C$ is $\Omega_{\text {push }(A)}$, so $\mathcal{V}=\left\{o_{A}\right\}$ and $\mathcal{C}=\left\{o_{A} \in\right.$ table $\}$. The first step is to generate a set of samples of $o_{A}$ in $R \cap$ table $=R$; poses $o_{A}=p_{4}$ and $o_{A}=p_{5}$ are such samples (note that 


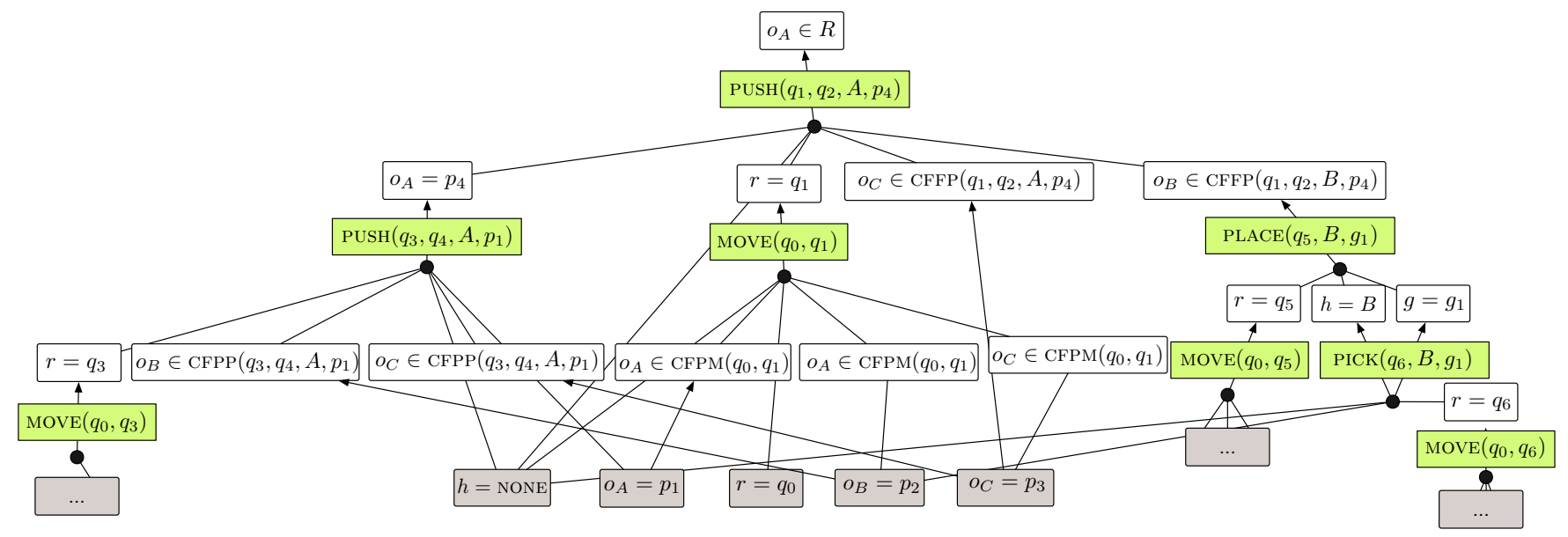

Fig. 5: Building the reachability graph.

we automatically reject sampled poses that would cause a collision with a permanent object), which we add to $G$. Next, we would use S-TRANSITION to generate samples $\left(o, a, o^{\prime}\right)$; however, because object $A$ cannot be grasped, there are no other coss that can be connected to $\Omega$. Finally, we use SROADMAP to generate samples $\left(o, a, o^{\prime}\right)$ that move within $\Omega_{\text {push }(A)}$. This generates several more vertices, shown as $o_{A}=p_{6}$ and $o_{A}=p_{7}$. The values of $a$ are complete ground PUSH action instances; we show two particular ones (that connect up to the initial pose of $\left.A, p_{1}\right)$ in the figure. We add the condition constraints of the new actions that cannot be satisfied using an existing vertex to the agenda, as well as putting the original item back on. At this point, the agenda is: $\left[\left(o_{B} \in \operatorname{CFPP}\left(q_{1}, q_{2}, A, p_{4}\right), \operatorname{PUSH}\left(q_{1}, q_{2}, A, p_{4}\right)\right)\right.$,

$\left(r=q_{1}, \operatorname{PUSH}\left(q_{1}, q_{2}, A, p_{4}\right)\right)$,

$\left(r=q_{3}, \operatorname{PUSH}\left(q_{3}, q_{4}, A, p_{1}\right)\right)$,

$\left(o_{A} \in R\right.$, goalSat)]. (CFPP is an abbreviation for C-FREEPOSES-PUSH.) The first constraint is that object $B$ not be in the way of the final push of $A$; the second two are that the robot be in the necessary configuration to perform each push.

\section{Iteration 2: From $Q$, we pop}

$C, a=\left(o_{B} \in \operatorname{CFPP}\left(q_{1}, q_{2}, A, p_{4}\right), \operatorname{PUSH}\left(q_{1}, q_{2}, A, p_{4}\right)\right)$. There are multiple COSs that have non-null intersection with $C$, and they would each be used to generate new vertices and edges for $G$. In our exposition, we will focus on $\Omega_{\text {place }(B)}$, so that $\mathcal{V}=\left\{o_{B}\right\}$ and $\mathcal{C}=\left\{o_{B} \in\right.$ table $\}$. The intersection of constraints is that $o_{B} \in \operatorname{CFPP}\left(q_{1}, q_{2}, A, p_{4}\right)$, and so we can generate samples of poses for $B$ that are not in the way of pushing $A$. Vertices like $o_{B}=p_{8}$ and $o_{B}=p_{9}$ are added to $G$. Next, we use S-TRANSITION to generate samples $\left(o, a, o^{\prime}\right)$ that connect from other coss; in particular, to move in from $\Omega_{p i c k(B)}$ we might sample action $\operatorname{PLACE}\left(q_{5}, B, g_{1}\right)$ where $p_{8}=\operatorname{POSE}\left(q_{5}, g_{1}\right)$. Finally, S-ROADMAP is unable to generate samples that move within $\Omega_{\text {place }(B)}$. At this point, we can add the PLACE action to $G$, as well as its conditions, and add its unmet conditions to the agenda. The agenda will be augmented with $\left(\left(r=q_{5}, \operatorname{PLACE}\left(q_{5}, B, g_{1}\right)\right),(h=\right.$ $\left.B, \operatorname{PlACE}\left(q_{5}, B, g_{1}\right)\right),\left(g=g_{1}, \operatorname{PLACE}\left(q_{5}, B, g_{1}\right)\right)$.
Subsequent iterations: In subsequent iterations, an instance of PICK for object $B$ will be added, which can satisfy both the $h=B$ and $g=g_{1}$ conditions of the PLACE action, and several MOVE actions will need to be added to satisfy the robot configuration conditions. Figure 5 shows a subgraph of $G$ containing one complete derivation of $\Gamma$. Nodes in gray with labels in them are vertices that are part of $s_{0}$. Three MOVE actions are shown with their condition constraints elided for space reasons, but they will be analogous to the one MOVE action that does have its conditions shown in detail. The green labels show the actions that are part of this derivation; there are 8 of them, which would yield an $\mathrm{H}_{\mathrm{FF}}$ value of 8 for $s_{0}$, unless there was a shorter derivation of $\Gamma$ also contained in $G$. But, in fact, the shortest plan for solving this problem involves 8 steps, so the heuristic is tight in this case. Additionally, all of the actions in $G$ whose conditions are satisfied in $s_{0}$ are applied by the forward search; so all four MOVE actions will be considered in the first iteration.

This example illustrates the power that the RG has in guiding the forward search; the cost of computing it is non-negligible, but it is able to leverage searches in lowdimensional spaces using state-of-the-art motion-planning algorithms very effectively. Two very important things happened in the process illustrated above. First, in planning how to push $A$ into the goal region, the system was able to first reason only about the object and later worry about how to get the robot into position to perform the pushing actions. Second, it was able to plan the pushes without worrying about the fact that object $B$ was in the way, and then later reason about how to clear the path. This style of backward goal-directed reasoning is very powerful and has been used effectively to solve NAMO (navigation among movable obstacle) problems [24] and by the HPN planner [18].

\section{EXPERIMENTAL RESULTS}

We applied HBF to six different manipulation problems to characterize its performance. The planner, samplers, and PR2 robot manipulation simulations were written in Python using 


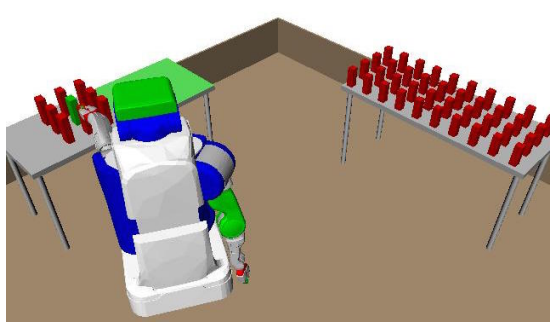

(a) Problem 1

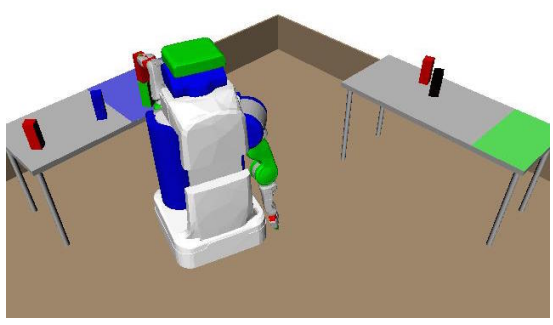

(d) Problem 4

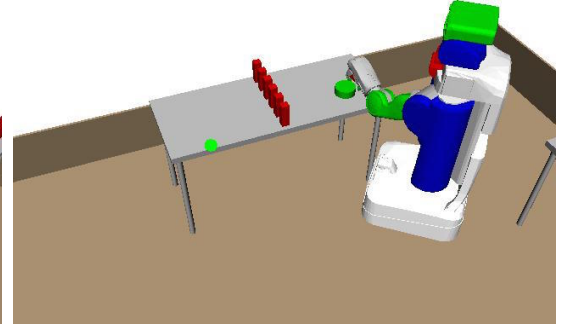

(b) Problem 2

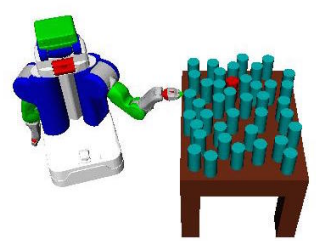

(e) Problem 5

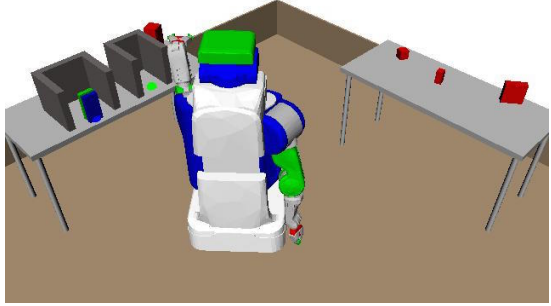

(c) Problem 3

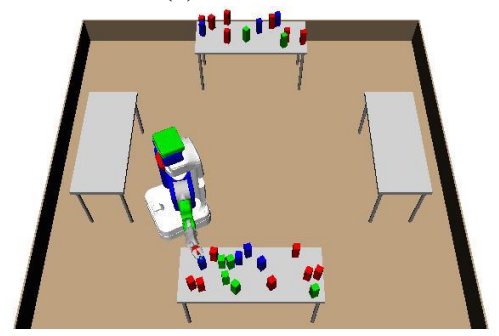

(f) Problem 6

Fig. 6: An early state on a valid plan for each problem.

\begin{tabular}{||c||c|c|c|c||c|c|c|c||}
\hline \multicolumn{1}{||c|}{$\mathrm{P}$} & \multicolumn{3}{|c||}{$\mathrm{H}_{0}$} & \multicolumn{4}{|c||}{$\mathrm{H}_{\mathrm{FF}}$} \\
\hline & $\%$ & runtime & length & visited & $\%$ & runtime & length & visited \\
\hline 1 & 100 & $12(7)$ & $8(0)$ & $312(280)$ & 100 & $4(1)$ & $12(2)$ & $12(4)$ \\
\hline 2 & 97 & $62(26)$ & $16(0)$ & $416(74)$ & 100 & $7(1)$ & $16(0)$ & $20(2)$ \\
\hline 3 & 62 & $238(62)$ & $16(0)$ & $4630(1424)$ & 100 & $6(1)$ & $16(0)$ & $74(4)$ \\
\hline 4 & 0 & $300(0)$ & $-(-)$ & $2586(186)$ & 97 & $12(4)$ & $24(4)$ & $112(48)$ \\
\hline 5 & 0 & $300(0)$ & $-(-)$ & $3182(762)$ & 98 & $23(9)$ & $24(4)$ & $170(74)$ \\
\hline 6 & 0 & $300(0)$ & $-(-)$ & $1274(80)$ & 100 & $82(13)$ & $72(4)$ & $382(74)$ \\
\hline
\end{tabular}

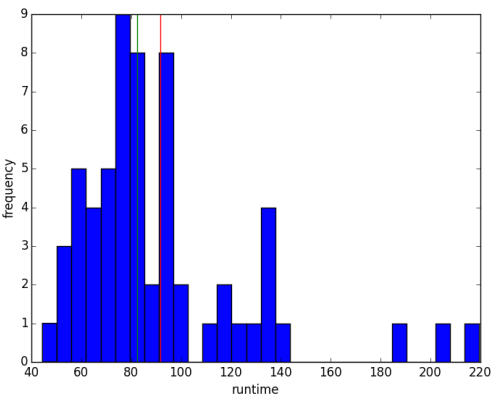

Fig. 7: Manipulation experiment results over 60 trials.

Fig. 8: Problem $6 \mathrm{H}_{\mathrm{FF}}$ runtimes.

OpenRAVE [25] without substantial performance optimization. In each problem, red objects represent movable objects that have no specified goal constraints. However, they impose geometric constraints on the problem and must usually be manipulated in order to produce a satisfying plan.

Problem 1: The goal constraint is for the green block, which is surrounded by 8 red blocks, to be on the green region on the table. Notice that the right table has 40 movable red objects on it that do not block the path of the green object.

Problem 2: The goal constraint is for the green cylinder to be at the green point on the edge of the table. The cylinder is too big for the robot to to grasp, so it must push it instead. The robot must move several of the red objects and then push the green cylinder several times to solve the problem.

Problem 3: A thin but wide green block starts behind a similar blue block. The goal constraints are that the green block be at the green point and that blue block be at the blue point, which is, in fact, its initial location. The is problem is non-monotonic in that the robot must first violate one of the goal constraints, and then re-achieve it. Additionally, the right cubby housing the green goal point is thinner than the left cubby, so the green block can only be placed using a subset of its grasps, all of which are infeasible for picking it up at its initial location. This forces the robot to place and regrasp the green block.

Problem 4: The goal constraints are that the green block be on the green region of the table, the blue block be on the blue region of the table, and the black block be on top of the blue block. Because the black block must end on the blue block, which itself must be moved, no static presampling of object poses would suffice to solve the problem. Additionally, a red block starts on top of the green block, preventing immediate movement of the green block.

Problem 5: This is exactly the same problem considered by Srivastava et al. [7]. The goal constraint is to be holding the red cylinder with an arbitrary grasp. 39 blue cylinders crowd the table, blocking the red object.

Problem 6: The goal constraints are that all 7 blue blocks must be on the left table and all 7 green blocks must be on the right table. There are also 14 red blocks. The close proximity of the blocks forces the planner to carefully order its operations as well as to move red blocks out of the way.

Solutions: The planner uses the same set of sampling primitives on each problem with no problem-dependent information. Each object has a possibly infinite number of grasps, computed from its geometry. However, the first four generated are typically sufficient to solve the problems 
posed here. We restrict the PR2 to side grasps to highlight the constrained nature of manipulation with densely packed objects.

We compared the performance of $\mathrm{HBF}$ when $\mathrm{H}=\mathrm{H}_{\mathrm{FF}}$ to the performance of an unguided version where $\mathrm{H}(s)=0$ for all $\mathrm{s}$. We enforce a five-minute timeout on all experiments. There were 60 trials per problem all conducted on a laptop with a $2.6 \mathrm{GHz}$ Intel Core $\mathrm{i} 7$ processor. Each entry in figure 7 reports the success percentage (\%) as well as the median and median absolute deviation (MAD) of the runtime, resulting plan length in terms of the number of actions, and number of states visited in the forward search. The median-based statistics are used to be robust against outliers. Figure 8 shows the distribution of runtimes for $\mathrm{H}_{\mathrm{FF}}$ on problem 6 . Several outliers cause the mean runtime of 93 (the red line) to be larger than the median runtime (the green line). The statistics for trials that failed to find a solution are included in the entries. Thus, entries with a runtime of 300 and MAD of 0 did not find a plan for any trial. The accompanying video simulates the PR2 executing a solution found by HBF for each problem.

Both versions of HBF solve problem 1 in several seconds despite the extremely high-dimensional configuration space. A manipulation planning algorithm that explores all adjacent modes [3], [4] would be overwhelmed by the branching factor despite the otherwise simple nature of the problem. The unguided search was unable to solve problems 4,5 , and 6 in under 5 minutes due to the size of the constructed state space and planning horizon. On problem 5, Srivastava et al. report a 63 percent success rate (over randomly generated initial conditions) with an average runtime of 68 seconds. The median runtime of HBF is about a third of the Srivastava et al. runtime with a 98 percent success rate. Problem 6 is comparable in nature to but more complex than task 5 considered in FFRob [20]. Additionally, the runtime is about half the FFRob reported median runtime of 157 seconds.

Conclusions: The experiments show that by leveraging the factored nature of common manipulation actions, HBF is able to efficiently solve complex manipulation tasks. The runtimes are improvements over runtimes on comparable problems reported by Srivastava et al. and Garrett et al. [20], [7]. Additionally, the dynamic search allows HBF to solve regrasping, pushing, and stacking problems all using the same planning algorithm. These results show promise of HBF scaling to the large and diverse manipulation problems prevalent in real world applications.

\section{REFERENCES}

[1] T. Siméon, J.-P. Laumond, J. Cortés, and A. Sahbani, "Manipulation planning with probabilistic roadmaps," IJRR, vol. 23, pp. 729-746, 2004.

[2] S. Cambon, R. Alami, and F. Gravot, "A hybrid approach to intricate motion, manipulation and task planning," International Journal of Robotics Research, vol. 28, 2009.

[3] K. Hauser and J. Latombe, "Integrating task and prm motion planning: Dealing with many infeasible motion planning queries," in ICAPSO9 Workshop on Bridging the Gap between Task and Motion Planning, 2009.
[4] K. Hauser and V. Ng-Thow-Hing, "Randomized multi-modal motion planning for a humanoid robot manipulation task," IJRR, vol. 30, no. 6, pp. 676-698, 2011.

[5] M. R. Dogar and S. S. Srinivasa, "A planning framework for nonprehensile manipulation under clutter and uncertainty," Auton. Robots, vol. 33, no. 3, pp. 217-236, 2012.

[6] J. Barry, L. P. Kaelbling, and T. Lozano-Perez, "A hierarchical approach to manipulation with diverse actions," in Robotics and Automation (ICRA), 2013 IEEE International Conference on. IEEE, 2013, pp. 1799-1806.

[7] S. Srivastava, E. Fang, L. Riano, R. Chitnis, S. Russell, and P. Abbeel, "Combined task and motion planning through an extensible plannerindependent interface layer," in IEEE Conference on Robotics and Automation (ICRA), 2014.

[8] T. Lozano-Pérez, "Automatic planning of manipulator transfer movements," IEEE Transactions on Systems, Man, and Cybernetics, vol. 11, pp. 681-698, 1981.

[9] T. Lozano-Pérez, J. L. Jones, E. Mazer, P. A. O’Donnell, W. E. L. Grimson, P. Tournassoud, and A. Lanusse, "Handey: A robot system that recognizes, plans, and manipulates," in IEEE International Conference on Robotics and Automation, 1987.

[10] G. T. Wilfong, "Motion planning in the presence of movable obstacles," in Symposium on Computational Geometry, 1988, pp. 279-288.

[11] R. Alami, T. Simeon, and J.-P. Laumond, "A geometrical approach to planning manipulation tasks. the case of discrete placements and grasps," in ISRR, 1990.

[12] R. Alami, J.-P. Laumond, and T.Siméon, "Two manipulation planning algorithms," in WAFR, 1994.

[13] C. Dornhege, P. Eyerich, T. Keller, S. Trüg, M. Brenner, and B. Nebel, "Semantic attachments for domain-independent planning systems," in International Conference on Automated Planning and Scheduling (ICAPS). AAAI Press, september 2009, pp. 114-121.

[14] C. Dornhege, A. Hertle, and B. Nebel, "Lazy evaluation and subsumption caching for search-based integrated task and motion planning," in IROS workshop on AI-based robotics, 2013.

[15] E. Erdem, K. Haspalamutgil, C. Palaz, V. Patoglu, and T. Uras, "Combining high-level causal reasoning with low-level geometric reasoning and motion planning for robotic manipulation," in IEEE International Conference on Robotics and Automation (ICRA), 2011.

[16] F. Lagriffoul, D. Dimitrov, A. Saffiotti, and L. Karlsson, "Constraint propagation on interval bounds for dealing with geometric backtracking," in IEEE/RSJ International Conference on Intelligent Robots and Systems (IROS), 2012.

[17] E. Plaku and G. Hager, "Sampling-based motion planning with symbolic, geometric, and differential constraints," in IEEE International Conference on Robotics and Automation (ICRA), 2010.

[18] L. P. Kaelbling and T. Lozano-Perez, "Hierarchical planning in the now," in IEEE Conference on Robotics and Automation (ICRA), 2011.

[19] A. K. Pandey, J.-P. Saut, D. Sidobre, and R. Alami, "Towards planning human-robot interactive manipulation tasks: Task dependent and human oriented autonomous selection of grasp and placement," in RAS/EMBS International Conference on Biomedical Robotics and Biomechatronics, 2012

[20] C. R. Garrett, T. Lozano-Pérez, and L. P. Kaelbling, "Ffrob: An efficient heuristic for task and motion planning," in International Workshop on the Algorithmic Foundations of Robotics (WAFR), 2014.

[21] B. Bonet and H. Geffner, "Planning as heuristic search: New results," in Proc. of 5th European Conf. on Planning (ECP), 1999, pp. 360-372.

[22] — , "Planning as heuristic search," Artificial Intelligence, vol. 129, no. 1, pp. 5-33, 2001.

[23] J. Hoffmann and B. Nebel, "The FF planning system: Fast plan generation through heuristic search," Journal Artificial Intelligence Research (JAIR), vol. 14, pp. 253-302, 2001.

[24] M. Stilman and J. J. Kuffner, "Planning among movable obstacles with artificial constraints," in Proceedings of the Workshop on Algorithmic Foundations of Robotics (WAFR), 2006.

[25] R. Diankov and J. Kuffner, "Openrave: A planning architecture for autonomous robotics," Robotics Institute, Carnegie Mellon University, Tech. Rep. CMU-RI-TR-08-34, 2008. 